Acta Crystallographica Section D

Biological

Crystallography

ISSN 0907-4449

\title{
Application of DEN refinement and automated model building to a difficult case of molecular- replacement phasing: the structure of a putative succinyl-diaminopimelate desuccinylase from Corynebacterium glutamicum
}

\begin{abstract}
Axel T. Brunger, ${ }^{a, b *}$ Debanu Das, ${ }^{\mathrm{c}, \mathrm{d}}$ Ashley M. Deacon, ${ }^{\mathrm{c}, \mathrm{d}}$ Joanna Grant, ${ }^{\text {c,e }}$ Thomas C. Terwilliger, ${ }^{\mathrm{f}}$ Randy J. Read, ${ }^{\mathrm{g}}$ Paul D. Adams, ${ }^{\text {h }}$ Michael Levitt and Gunnar F. Schröder'
\end{abstract}

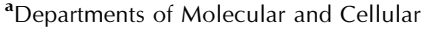
Physiology, Neurology and Neurological Sciences, and Photon Science, Stanford University, USA, ${ }^{\mathbf{b}}$ Howard Hughes Medical Institute, USA, 'Joint Center for Structural Genomics, USA, ${ }^{\mathbf{d}}$ Stanford Synchrotron Radiation Lightsource, SLAC National Accelerator Laboratory, USA, ${ }^{\mathbf{e} P \text { Protein Sciences }}$ Department, Genomics Institute of the Novartis Research Foundation, USA, '́tos Alamos National Laboratory, USA, ${ }^{\mathbf{g}}$ Department of Haematology, University of Cambridge, Cambridge Institute for Medical Research, England, ' Department of Bioengineering, University of California at Berkeley and Lawrence Berkeley National Laboratory, Berkeley, USA, 'Department of Structural Biology, Stanford University School of Medicine, USA, and 'institute of Complex Systems (ICS-6), Forschungszentrum Jülich, Germany
}

Correspondence e-mail: brunger@stanford.edu
Phasing by molecular replacement remains difficult for targets that are far from the search model or in situations where the crystal diffracts only weakly or to low resolution. Here, the process of determining and refining the structure of Cgl1109, a putative succinyl-diaminopimelate desuccinylase from Corynebacterium glutamicum, at $\sim 3 \AA$ resolution is described using a combination of homology modeling with MODELLER, molecular-replacement phasing with Phaser, deformable elastic network (DEN) refinement and automated model building using AutoBuild in a semi-automated fashion, followed by final refinement cycles with phenix.refine and Coot. This difficult molecular-replacement case illustrates the power of including DEN restraints derived from a starting model to guide the movements of the model during refinement. The resulting improved model phases provide better starting points for automated model building and produce more significant difference peaks in anomalous difference Fourier maps to locate anomalous scatterers than does standard refinement. This example also illustrates a current limitation of automated procedures that require manual adjustment of local sequence misalignments between the homology model and the target sequence.
Received 27 September 2011 Accepted 21 November 2011

PDB Reference:

succinyl-diaminopimelate desuccinylase, 3 tx8.

\section{Introduction}

Successful molecular-replacement phasing depends on a number of factors such as the proximity of the search model to the true structure, the quality and completeness of the diffraction data (especially at lower resolution), the solvent content, the presence of noncrystallographic symmetry and the limiting resolution $\left(d_{\min }\right)$ of the crystals. Although recent advances in reciprocal-space refinement such as deformable elastic network (DEN) refinement (Schröder et al., 2010), jelly-body refinement (Murshudov et al., 2011) and real-space refinement (DiMaio et al., 2011) enable structure determination from more distant models, the ultimate success of molecular replacement phasing depends on whether previously unknown parts of the model become visible in the electrondensity maps or whether conformational changes in the structure are uniquely determined.

DEN refinement consists of torsion-angle refinement interspersed with $B$-factor refinement in the presence of a 
sparse set of distance restraints (typically one per atom, randomly selected) which are initially obtained from a reference model (Schröder et al., 2010). The reference model can simply be the starting model for refinement or it can be a homology or predicted model that provides external information. During the process of torsion-angle refinement with a slow-cooling simulated-annealing schema, the DEN distance restraints are adjusted in order to fit the diffraction data. The degree of this adjustment or deformation of the initial distance restraints is controlled by a parameter $\gamma$. The method of jelly-body refinement (Murshudov et al., 2011) bears some resemblance to the special case of DEN refinement with $\gamma=1$. The weight of the DEN distance restraints is controlled by another parameter, $w_{\mathrm{DEN}}$. A two-dimensional grid search for $\left(\gamma, w_{\text {DEN }}\right)$ is performed in which multiple refinements for each parameter pair are performed with different initial randomnumber seeds for the velocity assignments of the torsion-angle molecular-dynamics method and different randomly selected DEN distance restraints. The globally optimal model (in terms of minimal $R_{\text {free }}$, possibly assisted by geometric validation criteria) is then used for further refinement and model building. By default, the last two macrocycles of the DEN refinement protocol are performed without any DEN restraints, so the resulting model is not strained or biased by the reference model (although such restraints can be useful at very low resolution). In other words, the DEN restraints guide the refinement path, increasing the chances of obtaining a better model than with standard refinement. In addition, the deformability of the DEN restraints makes this method more general than rigid-body or normal-mode refinement. Thus, DEN refinement is a general refinement method that can be applied to any starting model and reference model. In practice, the reference model is likely to be identical to the starting model. However, there are situations in which the reference model can be different from the starting model. For example, re-refinements of existing structures can be performed using structures of homologous proteins that were not available at the time the original structure was determined.

A number of highly automated procedures for model building and model rebuilding have recently been developed (Levitt, 2001; Oldfield, 2002, 2003; Ioerger \& Sacchettini, 2003; DePristo et al., 2005; Cowtan, 2006; Langer et al., 2008; Terwilliger et al., 2008). A key feature of several of these procedures is alternation between model building and calculation of electron-density maps. Each local improvement in the model leads to an overall improvement in the map, which in turn makes additional improvements in the model possible. In this work, we use one of these procedures, the AutoBuild method (Terwilliger et al., 2008) as implemented in PHENIX (Adams et al., 2010), as a core tool for model improvement. In one cycle of model rebuilding with AutoBuild, a densitymodified electron-density map is calculated beginning with phases from the working model and including any available experimental phase information. A new model is then built and refined with phenix.refine (Afonine et al., 2005). Two methods for rebuilding the working model are used here. In the first method, several new models (or segments) are built without reference to the working model. The parts of the new models and the working model that best fit the electrondensity map are then merged together to form a composite model. Using this procedure, the model can change in any way during rebuilding. In the second method, termed 'rebuilding in place', segments of the working model are rebuilt one at a time, maintaining connectivity and sequence alignment. This 'rebuilding-in-place' procedure therefore adjusts the position of existing atoms in the structure and can be thought of as an extension of refinement.

In this paper, we describe the process of determining the crystal structure of Cgl1109 (Joint Center for Structural Genomics target 376512 listed in TargetDB; http:// targetdb.sbkb.org/TargetDB/), a putative succinyl-diaminopimelate desuccinylase from Corynebacterium glutamicum, using a combination of molecular-replacement phasing, refinement and semi-automated model building. At the later stages, experimental phase information from SeMet MAD phasing was included in the refinement. It should be noted that these MAD phases were of insufficient quality to allow automated model building, and manual building would have been exceedingly difficult and time-consuming even for a highly skilled crystallographer (see §3.6). Thus, molecularreplacement phasing was attempted. However, manual interpretation of the initial electron-density map again proved difficult. Indeed, Cgl1109 was one of the cases used to test the performance of real-space refinement of the molecularreplacement solution in conjunction with the Rosetta empirical energy function (DiMaio et al., 2011; case 10 in Table 1 in this reference), but the refinement was not completed owing to poor or disordered density in numerous regions and low resolution $\left(R_{\text {free }}=0.39\right.$; Table 1 in DiMaio et al., 2011).

Here, we present an independent structure determination of Cgl1109 at $\sim 3 \AA$ resolution without use of the previous Rosetta model and molecular-replacement solution. A homology model of Cgl1109 was created using sequence alignment with PROMALS3D (Pei et al., 2008) and modeling with MODELLER (Sali \& Blundell, 1993) starting from the structure of succinyl-diaminopimelate desuccinylase from the $\beta$-proteobacterium Neisseria meningitidis (PDB entry 1vgy; Badger et al., 2005). The structure was determined by molecular replacement with Phaser (McCoy et al., 2007) using a model edited with Sculptor (Bunkóczi \& Read, 2011), followed by DEN refinement with a full $\left(\gamma, w_{\text {DEN }}\right)$ grid search (Schröder et al., 2010), automated model building with AutoBuild, determination of the selenium sites by anomalous difference Fourier maps, calculation of MAD phase probability distributions using a maximum-likelihood method (Burling et al., 1996) and completion of the refinement in a semi-automated fashion using AutoBuild and phenix.refine (Adams et al., 2010) with the MLHL target function (Pannu et al., 1998). The final model has excellent geometry and $R_{\text {cryst }}$ and $R_{\text {free }}$ values of 0.238 and 0.257 , respectively, at $2.97 \AA$ resolution.

This example shows that DEN refinement with a full $\left(\gamma, w_{\mathrm{DEN}}\right)$ grid search generally produces models that are 
closer to the true structure than standard (gradient-descent) or simulated-annealing refinement methods, resulting in improved model phases and better $R$ values. The improved model phases in turn provide better starting points for automated model building with AutoBuild. This approach ultimately produced a well refined structure that would have been very difficult to achieve with manual model building and standard refinement. Moreover, the improved model phases produce more significant difference peaks that better locate the anomalous diffraction selenium sites. Compared with the Rosetta refinement method (DiMaio et al., 2011), DEN refinement has the advantage that it does not require extensive empirical energy-function simulations and that it has been shown to also work well for structures determined at low resolution (worse than $3.5 \AA$ ). The successful application to Cgl1109 demonstrates that DEN refinement also has significant utility for structures determined at $\sim 3 \AA$ resolution, especially for cases of anisotropic diffraction and/or high $B$ factors. The research performed in this paper also serves as a tutorial for the combined use of various methods and computer software systems to tackle difficult molecularreplacement cases. The corresponding data files have been made available on the $C N S$ website in the tutorial section for DEN refinement.

\section{Materials and methods}

\subsection{Crystallization}

Cgl1109 was expressed, purified and crystallized using the JCSG high-throughput structural biology pipeline (Elsliger $e t$ al., 2010) and standard JCSG protocols with crystallization modifications. Briefly, clones were generated using the Polymerase Incomplete Primer Extension (PIPE) cloning method (Klock et al., 2008). The gene encoding Cgl1109 (GenBank NP_600337, gi|19552335; UniProt Q59284) was PCR-amplified from C. glutamicum 534 genomic DNA using PfuTurbo DNA polymerase (Stratagene) and I-PIPE primers (forward primer, $5^{\prime}$-ctgtacttccagggcCTGTACTTCCAGGGCATGAACTCTGAACTCAAACCAGGATTAG-3'; reverse primer, 5'-aattaagtcgcgttaAATTAAGTCGCGTTACTCGCTCAGGTACTGCTTCAAAATTGC- $3^{\prime}$; target sequence in upper case) that included sequences for the predicted $5^{\prime}$ and $3^{\prime}$ ends. The genomic DNA used here and obtained from the American Type Culture Collection (ATCC) contained two amino-acid substitutions (Glu4Asn and Lys6Gln) and one amino-acid deletion (Leu5), as confirmed by DNA sequencing, when compared with the available GenBank sequence from C. glutamicum 534; these mutations are unlikely to affect the biochemical properties of the enzyme based on their locations. Expression was performed in selenomethionine-containing medium at $298 \mathrm{~K}$. Selenomethionine was incorporated via inhibition of methionine biosynthesis (Van Duyne et al., 1993), which does not require a methionine-auxotrophic strain. The protein was purified by two steps of nickel-chelating chromatography (GE Healthcare) with an intermediate step involving TEV protease cleavage of the purification tag and was concentrated to $18.5 \mathrm{mg} \mathrm{ml}^{-1}$ by centrifugal ultrafiltration (Millipore) for crystallization trials. Crystals used for structure determination were grown using Microseed Matrix Screening (MMS; Ireton \& Stoddard, 2004; D'Arcy et al., 2007) as implemented with an Oryx8 crystallization robot (Douglas Instruments). Initial seed crystals used for MMS were grown using the nanodroplet vapor-diffusion method from sitting drops composed of $200 \mathrm{nl}$ protein solution mixed with $200 \mathrm{nl}$ crystallization solution equilibrated against a $50 \mu \mathrm{l}$ reservoir at $293 \mathrm{~K}$ for 48 days prior to harvest. The crystals used for the seed stock were obtained using a precipitating reagent consisting of $0.2 \mathrm{M} \mathrm{MgCl}_{2}, 30 \%$ PEG 400, 0.1 M HEPES pH 7.5. The entire crystallization drop $(400 \mathrm{nl})$ containing the seed crystals was aspirated using a pipette and placed in a Seed Bead tube (Hampton Research) stored on ice. To ensure that all crystals were transferred to the Seed Bead tube, the empty shelf was rinsed with $50 \mu \mathrm{l}$ mother liquor. The Seed Bead tube containing the seed stock was vortexed vigorously for three intervals of $30 \mathrm{~s}$, keeping the tube on ice between each vortex. Final MMS crystallization plates were set up on the Oryx8 as sitting drops composed of $150 \mathrm{nl}$ protein, $100 \mathrm{nl}$ crystallization solution and $50 \mathrm{nl}$ seed stock. The final crystals used for structure determination were obtained from a crystallization reagent consisting of $43.1 \%$ polyethylene glycol $400,0.2 \mathrm{M}$ sodium chloride, $0.1 \mathrm{M}$ sodium/potassium phosphate $\mathrm{pH} 6.41$ at $293 \mathrm{~K}$ for $21 \mathrm{~d}$ prior to harvest. $6 \mathrm{mM} \mathrm{ZnCl} 2$ was added to the protein prior to setup. No additional cryoprotectant was added to the crystal. Initial screening for diffraction was carried out using the Stanford Automated Mounting system (SAM; Cohen et al., 2002) at the Stanford Synchrotron Radiation Lightsource (SSRL; Menlo Park, California, USA).

\subsection{X-ray data collection, processing, structure validation and deposition}

MAD data were collected on beamline 9-2 at the SSRL at wavelengths corresponding to the high-energy remote $\left(\lambda_{1}\right)$, inflection point $\left(\lambda_{2}\right)$ and peak $\left(\lambda_{3}\right)$ wavelengths of a selenium MAD experiment using the Blu-Ice (McPhillips et al., 2002) data-collection environment. The data sets were collected at $100 \mathrm{~K}$ using a MAR Mosaic $325 \mathrm{CCD}$ detector (Rayonix, USA). The MAD data were integrated and reduced using $X D S$ (Kabsch, 2010) and scaled with XSCALE (Kabsch, 2010). Diffraction data and refinement statistics are summarized in Table 1 . The quality of the crystal structure was analyzed using the JCSG Quality Control server (http:// smb.slac.stanford.edu/jcsg/QC), which verifies the stereochemical quality of the model using AutoDepInputTool (Yang et al., 2004), MolProbity (Chen et al., 2010) and WHAT IF v.5.0 (Vriend, 1990); the agreement between the atomic model and the data using SFCHECK v.4.0 (Vaguine et al., 1999) and RESOLVE (Terwilliger, 2000); the protein sequence using ClustalW (Thompson et al., 1994); atom occupancies using MOLEMAN2 (Kleywegt, 2000) and the consistency of NCS pairs; and evaluates $R_{\text {free }} / R_{\text {cryst }}$ and the maximum/minimum $B$ factors. Atomic coordinates and experimental data for 
Table 1

Crystallographic data and refinement statistics for Cgl1109.

Values in parentheses are for the highest resolution shell.

\begin{tabular}{|c|c|c|c|}
\hline & $\begin{array}{l}\lambda_{1} \text { MAD-Se } \\
\text { (remote) }\end{array}$ & $\begin{array}{l}\lambda_{2} \text { MAD-Se } \\
\text { (inflection point) }\end{array}$ & $\begin{array}{l}\lambda_{3} \text { MAD-Se } \\
\text { (peak) }\end{array}$ \\
\hline Space group & \multicolumn{3}{|c|}{$P 6_{5} 22$} \\
\hline Unit-cell parameters $(\AA)$ & \multirow{2}{*}{\multicolumn{3}{|c|}{$a=82.90, b=82.90, c=364.18$}} \\
\hline \multicolumn{2}{|l|}{ Data collection } & & \\
\hline Wavelength $(\AA)$ & 0.9116 & 0.9794 & 0.9792 \\
\hline Resolution range $(\AA)$ & $29.5-2.97(3.05-2.97)$ & $29.5-3.17(3.26-3.17)$ & $29.5-2.97(3.05-2.97)$ \\
\hline No. of observations & 73623 & 60577 & 111259 \\
\hline No. of unique reflections & 16179 & 13404 & 16192 \\
\hline Completeness (\%) & $99.1(98.5)$ & $99.1(99.0)$ & $99.2(98.4)$ \\
\hline Mean $I / \sigma(I)$ & $13.1(1.5)$ & $14.9(2.8)$ & $17.2(1.7)$ \\
\hline$R_{\text {merge }}$ on $I^{\dagger}(\%)$ & $9.5(124.8)$ & $9.1(64.7)$ & $10.6(150.6)$ \\
\hline$R_{\text {meas }}$ on $I \$(\%)$ & $10.1(140.6)$ & $10.4(72.9)$ & $11.4(162.5)$ \\
\hline \multicolumn{4}{|l|}{ Model and refinement statistics } \\
\hline Resolution range $(\AA)$ & $29.5-2.97$ & & \\
\hline No. of reflections (total) & $16098 \S$ & & \\
\hline No. of reflections (test set) & 1649 & & \\
\hline Completeness $(\%)$ & 99.07 & & \\
\hline Data set used in refinement & $\lambda_{1} \mathrm{MAD}-\mathrm{Se}$ & & \\
\hline Cutoff criterion & $|F|>0$ & & \\
\hline$R_{\text {cryst }}$ 的 & 0.238 & & \\
\hline$R_{\text {free }} \uparrow$ & 0.257 & & \\
\hline \multicolumn{4}{|l|}{ Stereochemical parameters } \\
\hline \multicolumn{4}{|l|}{ Restraints (r.m.s.d. observed) } \\
\hline Bond angles $\left({ }^{\circ}\right)$ & 0.625 & & \\
\hline Bond lengths $(\AA)$ & 0.003 & & \\
\hline $\begin{array}{l}\text { Average protein isotropic } \\
B \text { factor }\left(\AA^{2}\right)\end{array}$ & $99.7+\dagger$ & & \\
\hline $\begin{array}{l}\text { Maximum-likelihood-based } \\
\text { coordinate error }(\AA)\end{array}$ & 0.71 & & \\
\hline Protein residues & 360 & & \\
\hline Phosphates/chlorides & $1 / 1$ & & \\
\hline
\end{tabular}

$\dagger R_{\text {merge }}=\sum_{h k l} \sum_{i}\left|I_{i}(h k l)-\langle I(h k l)\rangle\right| / \sum_{h k l} \sum_{i} I_{i}(h k l)$ (Diederichs \& Karplus, 1997) $\ddagger R_{\text {meas }}$ (redundancy-independent $\left.R_{\text {merge }}\right)=\sum_{h k l}\{N(h k l) /[N(h k l)-1]\}^{1 / 2} \sum_{i}\left|I_{i}(h k l)-\langle I(h k l)\rangle\right| / \sum_{h k l} \sum_{i} I_{i}(h k l)$. \& Typically, the number of unique reflections used in refinement is slightly less than the total number that were integrated and scaled. Reflections are excluded owing to negative intensities and rounding errors in the resolution limits and unit-cell parameters. $-R_{\text {cryst }}=$ $\sum_{h k l}|| F_{\text {obs }}|-| F_{\text {calc }}|| / \sum_{h k l}\left|F_{\text {obs }}\right|$, where $F_{\text {calc }}$ and $F_{\text {obs }}$ are the calculated and observed structure-factor amplitudes, respectively. $R_{\text {free }}$ is the same as $R_{\text {cryst }}$, but calculated using $10.24 \%$ of the total reflections that were chosen at random and omitted from refinement. $\dagger \dagger$ This value represents the total $B$, which includes overall TLS refinement and residual $B$ components.

Cgl1109 from C. glutamicum to $2.97 \AA$ A resolution (PDB entry 3tx8) have been deposited in the Protein Data Bank (http:// www.wwpdb.org).

\subsection{Homology modeling, structure determination and refinement}

PROMALS3D (Pei et al., 2008) was used for primarysequence alignment, MODELLER (Sali \& Blundell, 1993) was used for profile generation and homology modeling, Sculptor (Bunkóczi \& Read, 2011) and Phaser (McCoy et al., 2007) were used for molecular-replacement phasing, $C N S$ v.1.3 was used for DEN refinement (Schröder et al., 2010), AutoBuild (Terwilliger et al., 2008) was used for automated model building, Coot (Emsley et al., 2010) was used for manual rebuilding and structure validation, $C N S$ was used for MAD phasing and density modification (Brünger et al., 1998), phenix.refine (Adams et al., 2010) was used for final refinement cycles and PyMOL (DeLano, 2002) was used for molecular illustrations and structure and electron-density map superposition.

\section{Results and discussion}

\subsection{Search for similar structures, primary-sequence alignment and homology modeling}

A profile of structures related to the genomic sequence of Cgl1109 (Fig. 1) was generated using the MODELLER build_profile.py script (http:// www.salilab.org/modeller/tutorial/basic. html) and the current protein database file pdb_95.pir (updated 24 February 2011) available in the supplementary file download section of the MODELLER website. This produced a list of eight homologous structures (PDB entries 1cg2, 3ct9, 2f7v, 3gb0, 3isz, 3 pfo, 2rb7 and 1vgy) with sequence identities that varied between 24 and $28 \%$. A cluster analysis of these structures using the MODELLER compare.py script revealed that they are all relatively equidistant from each other, with the exception of PDB entries 3isz and 1vgy, which are closer to each other than to the other structures. Since there is no significant difference in terms of sequence identity to the target structure among these candidate models, the one with the highest resolution and best $R_{\text {free }}$ value was chosen for all further calculations (PDB entry 1 vgy chain $A$, referred to as $1 \mathrm{vgy}-A$ in the following), which was also the template used for Rosetta-based molecular replacement (DiMaio et al., 2011).

The success of molecular replacement depends on optimal sequence alignment between homologous structure and target sequence (Schwarzenbacher et al., 2004; Bunkóczi \& Read, 2011). To make some use of the structural information in the primary-sequence alignment we used the PROMALS3D program (Pei et al., 2008), resulting in the alignment shown in Fig. 1. PROMALS3D can produce more accurate sequence alignments compared with methods that do not make use of secondary-structure information for sequence pairs with at least $20 \%$ identity (Pei et al., 2008). Other methods such as HHpred (Söding, 2005) that include secondary-structure information may provide alternative alignments (see $\S 4$ ).

The primary-sequence alignment obtained with PROMALS3D and the structure of $1 \mathrm{vgy}-A$ were used as input for the generation of a homology model using the model-single.py script of MODELLER. All default parameters were used except that the a.very_fast() option was specified to perform a limited amount of target-function optimization with conjugate-gradient minimization. This limited amount of energy minimization keeps the resulting homology model closer to the crystal structure of $1 \operatorname{vgy}-A$, 
The MODELLER search model (with $B$ factors set to a uniform value of $50 \AA^{2}$ ) was first edited using Sculptor (Bunkóczi \& Read, 2011) with the PROMALS3D alignment (Fig. 1) in order to trim surface side chains (as suggested by Schwarzenbacher et al., 2004) and to modify the $B$ factors of the search model according to sequence similarity between Cgl1109 and 1vgy- $A$ (the similarity score was used for the $B$-factor modeling and the Schwarzenbacher score was used for the pruning). After clustering of the rotation-function and translation-function peaks and purging peaks below a $75 \%$ threshold (default settings in Phaser), a single solution emerged with RFZ $=3.2, \mathrm{TFZ}=9.9, \mathrm{LLG}=75, R_{\text {cryst }}=0.65$ and 11 clashes. The position and orientation of this solution was very similar to that obtained with molecular replacement using the 1vgy- $A$ search model, lending credence to the correctness of the solution. Furthermore, the solution was determined to be identical to that found by molecular replacement with the Rosetta search model (DiMaio et al., 2011) apart from application of symmetry and lattice operators. However, Phaser was unable to produce the correct solution when using a fully optimized model obtained with the default settings in MODELLER [as opposed to the minimal a.very_fast() setting]; inspection of the optimized MODELLER model revealed that it had significantly moved

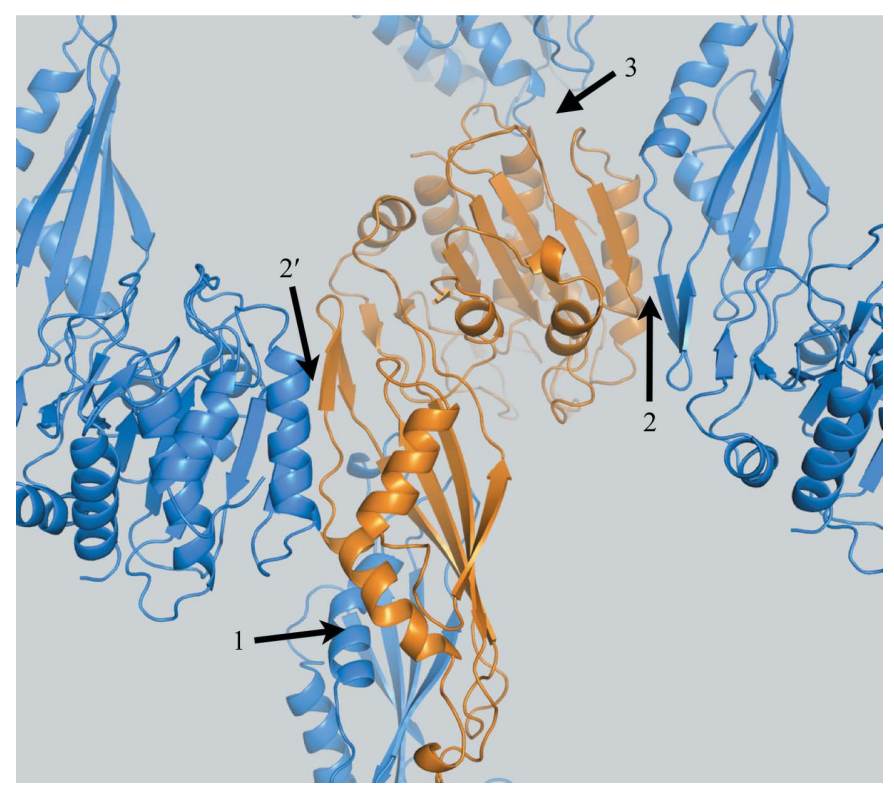

\section{Figure 2}

Interaction between symmetry-related molecules. A primary molecule (orange) and the nearest symmetry-related molecules (blue) obtained by applying the symmetry operators of the space group of the crystal $\left(P 6_{5} 22\right)$ to the primary molecule are shown, as well as lattice translations. Taken together, all these molecules form a network of interactions which is connected throughout the crystal in all three dimensions. The molecules interact through three interfaces, labelled 1, 2 and 3. Interface $2^{\prime}$ is related by crystallographic symmetry to interface 2 . Of the three interfaces, interface 1 involves the most extensive interactions, with a buried suface area of $1569 \AA^{2}$ (compared with $541 \AA^{2}$ for interface 2 and $276 \AA^{2}$ for interface 3 ; the buried surface areas were computed with the PDBePISA server). Considering the extensive interactions, interface 1 is likely to promote dimerization of the molecule, as is also suggested by the PDBePISA server. away from the $1 \mathrm{vgy}-A$ template and thus was apparently too distant from the true structure of Cgl1109 to produce a molecular-replacement solution. This example shows that it is useful to try different homology models and to score them according to the criteria provided by the particular molecularreplacement method used, e.g. rotation-function and translation-function $Z$ scores and log-likelihood gain in Phaser. In general, it is advisable to try additional searches in which the search model is broken up into subdomains that may exhibit different relative orientations and translations. However, this was unnecessary for $\mathrm{Cgl1109}$ as the subdomain placements were very similar between Cgl1109 and 1vgy- $A$ (see below).

A further validation of a molecular-replacement solution is provided by the overall crystal-packing arrangement and connectivity of the arrangement, i.e. no empty spaces should be left between the layers of molecules. Fig. 2 illustrates the connectivity of the arrangement and the three different interfaces that are created by symmetry and lattice operators.

\subsection{DEN refinement}

DEN refinement generally requires a starting model that matches the primary sequence of the target structure. There-

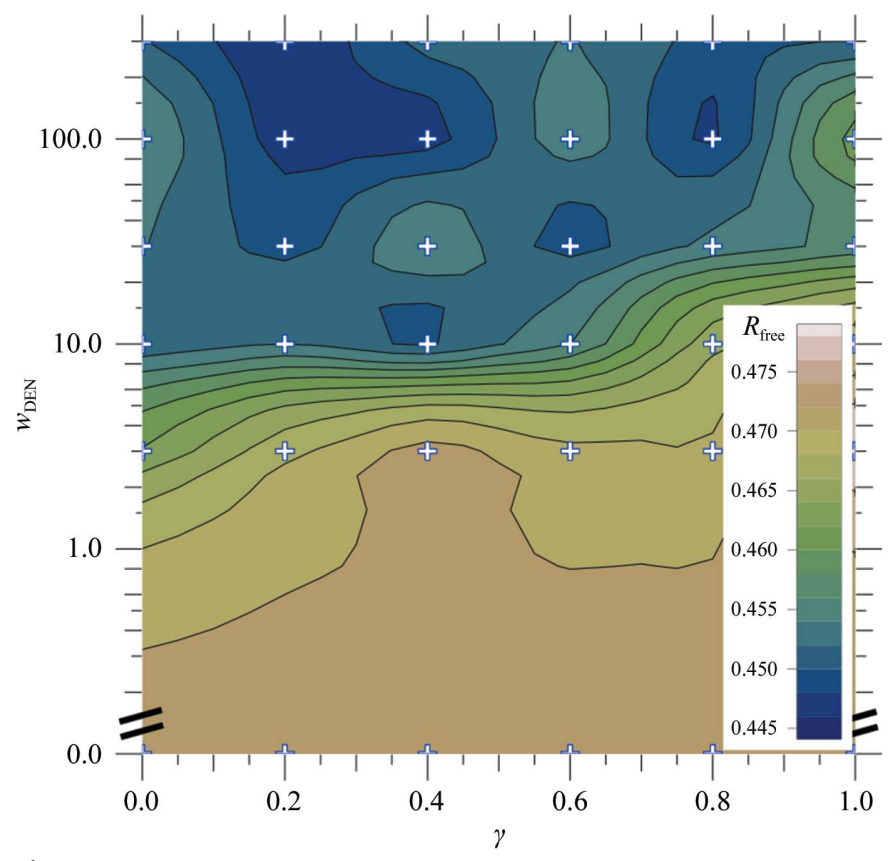

Figure 3

DEN refinement starting from molecular-replacement solution. The best $R_{\text {free }}$ value for each parameter pair $\left(\gamma, w_{\text {DEN }}\right)$ among 20 repeats is shown; for each parameter pair we performed 20 repeats of the DEN-refinement protocol consisting of ten macrocycles of torsion-angle refinement and restrained individual $B$-factor refinement (for details, see text). The $R_{\text {free }}$ value is contoured using values calculated on a $6 \times 6$ grid (marked by small + signs) where the parameter $\gamma$ is $(0.0,0.2,0.4,0.6,0.8,1.0)$ and $w_{\mathrm{DEN}}$ is $(0,3,10,30,100,300)$; the results for $w_{\mathrm{DEN}}=0$ (i.e. torsion-angle refinement without DEN restraints) are independent of $\gamma$ and the same value was used for all grid points with $w_{\mathrm{DEN}}=0$. The value of $R_{\text {free }}$ varies from 0.444 to 0.479 . The contour plot shows two pronounced minima in the range $300 \geq w_{\mathrm{DEN}} \geq 100$, with the absolute minimum at $w_{\mathrm{DEN}}=300$, $\gamma=0.2$. 
fore, the molecular-replacement solution obtained from the minimally optimized MODELLER model was used as the starting point for DEN refinement. Side chains that were pruned by Sculptor were added back to the model by superimposing the complete model obtained by MODELLER on the Phaser molecular-replacement solution. All $B$ factors were reset to a uniform value $\left(50 \AA^{2}\right)$. The resulting coordinates

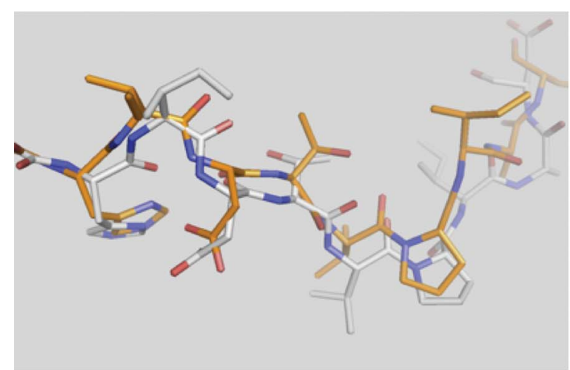

(a)

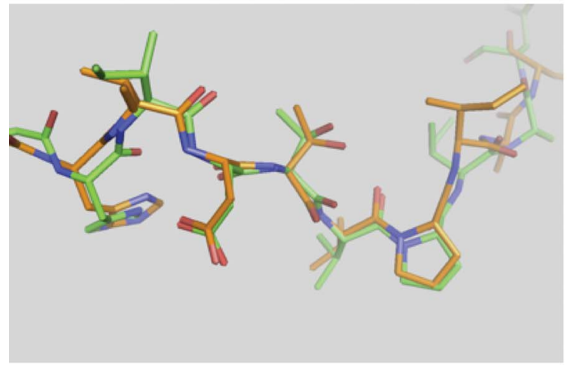

(c)

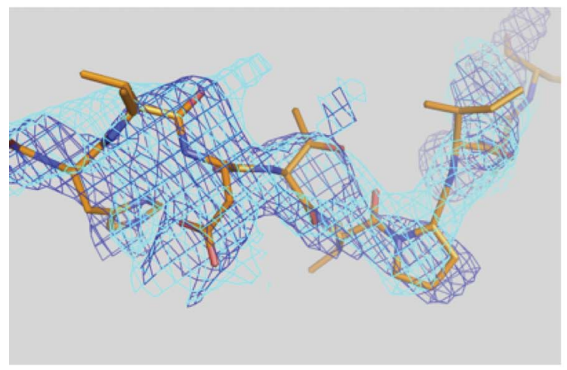

(e)

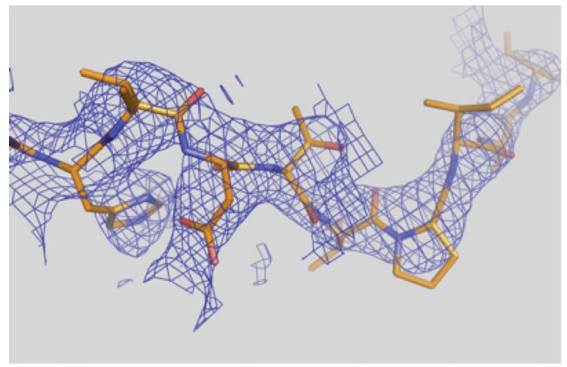

(g)

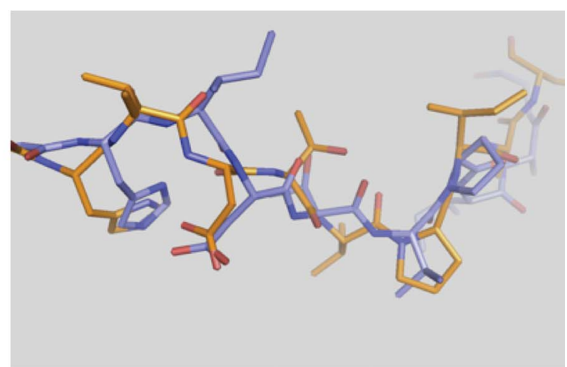

(b)

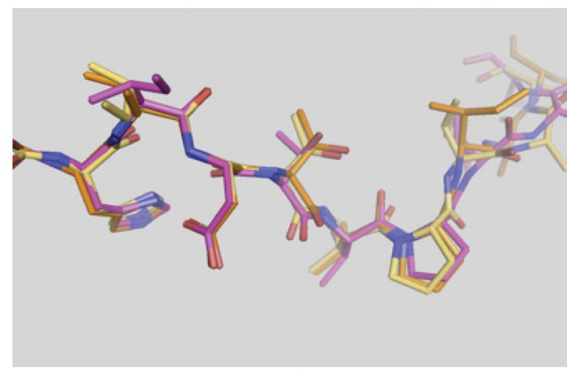

(d)

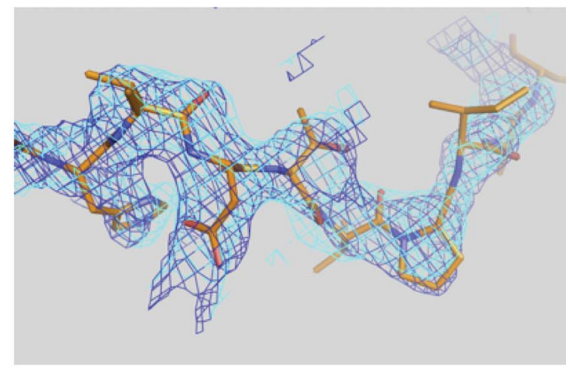

$(f)$

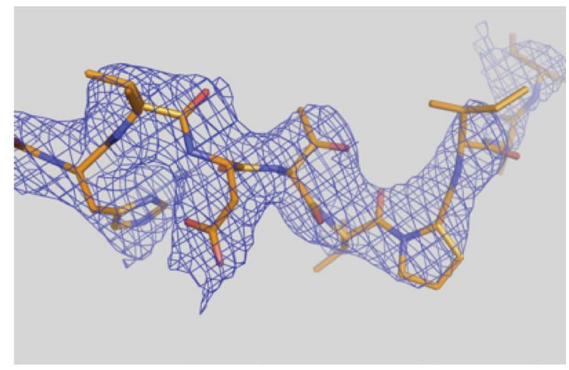

(h)
Figure 4

Comparison of various refinements and maps for residues 66-77. The sequence numbers refer to the genomic sequence of Cgl1109 (see Fig. 1). (a) Standard refinement (gray) versus the final model (orange). (b) Standard refinement and one round of AutoBuild (blue) versus the final model (orange). (c) DEN refinement (green) versus the final model (orange sticks). (d) DEN refinement and one round of AutoBuild (magenta) versus the result of semi-automated rebuilding (yellow) versus the the final model (orange). (e) $2 m F_{\mathrm{o}}-D F_{\mathrm{c}}$ electron-density map after standard refinement (blue mesh) and a subsequent round of AutoBuild (cyan mesh) versus the final structure (orange sticks). $(f) 2 m F_{\mathrm{o}}-D F_{\mathrm{c}}$ electron-density map after DEN refinement (blue) and a subsequent round of AutoBuild (cyan) versus the final structure (orange sticks). ( $g$ ) Electron-density map obtained by density modification of the MAD map (blue) versus the final structure (orange sticks). (h) $2 m F_{\mathrm{o}}-D F_{\mathrm{c}}$ electron-density map (blue mesh) of the final model (orange sticks). were used as both the starting and reference model for DEN (Schröder et al., 2010). The refinement protocol CNS v.13; het/Cns restrained individual $B$-factor refinement was carried out group $B$-factor refinement as appropriate for the resolution of Cgl1109. Specifically, ten macrocycles of torsion-angle refinement and restrained individual $B$ factor refinement were performed in which the first cycle always used $\gamma=0$, the following seven cycles used a specified value for $\gamma$ (see below) and the last two cycles were performed without DEN restraints. The MLF target function (Pannu \& Read, 1996) was used for the refinement against the diffraction data at the inflection point (the same diffraction data that were used in the work by DiMaio et al., 2011). In the final stages of refinement, the diffraction data at the high-energy remote wavelength were used (see below).

DEN distance restraints were generated from $N$ randomly selected pairs of atoms in the reference model that were separated by not more than ten residues along the polypeptide sequence and were separated by $3-15 \AA$ in space (default settings for DEN refinement in $C N S$ ). The value of $N$ was chosen to be equal to the number of atoms, so the set of distance restraints was relatively sparse, with an average of one restraint per atom.

We determined the optimum values of the $\gamma$ and $w_{\text {DEN }}$ parameters of DEN refinement by a global two-dimensional grid search (Fig. 3). At each grid point, 20 refinement repeats were performed with different random initial velocities and different randomly selected DEN distances. We used 30 combinations of six $\gamma$ values $(0.0,0.2,0.4,0.6,0.8$ and $1.0)$ and five $w_{\text {DEN }}$ values $(3,10,30,100$ and 300); we also included 20 repeats with $w_{\mathrm{DEN}}=0$ (corresponding to using the refinement protocol without DEN restraints, with the results being independent of $\gamma$ ). Of all the resulting models, the one with the lowest $R_{\text {free }}$ value (0.444; Fig. 3 and Table 1) was used for subsequent model building and refinement. Generally, if there are multiple models with similar low $R_{\text {free }}$ 
values, one could choose the one with the better geometry. The resulting model was substantially better in many places

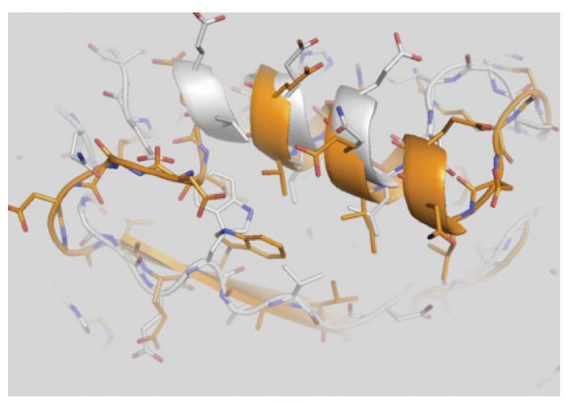

(a)

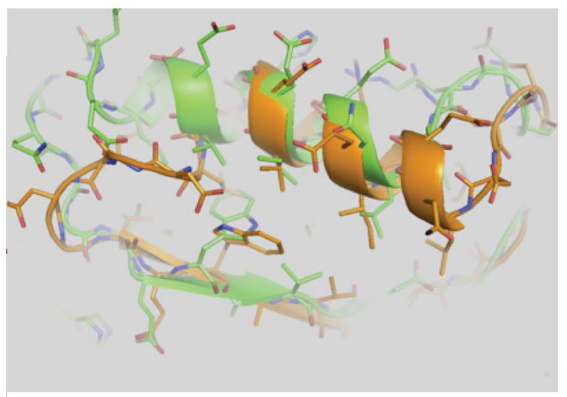

(c)

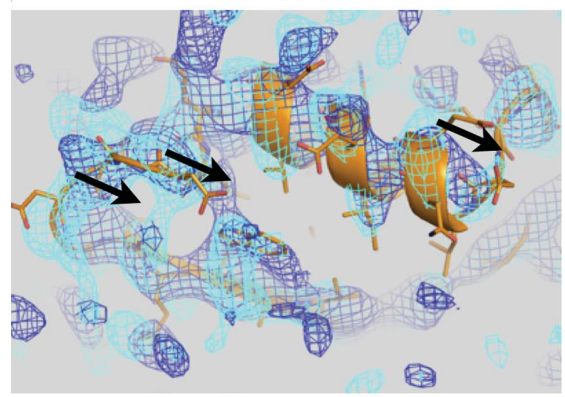

(e)

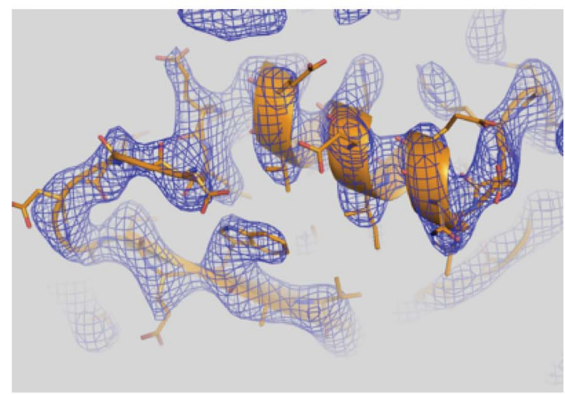

$(g)$

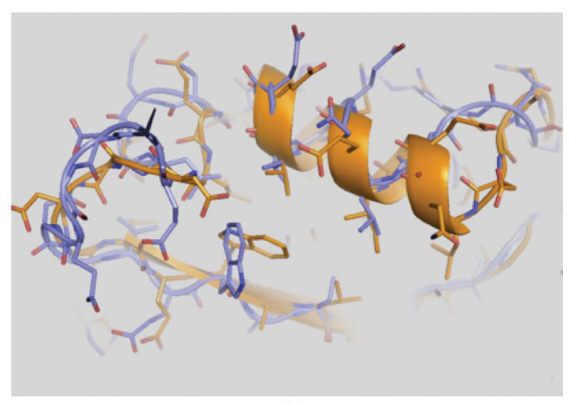

(b)

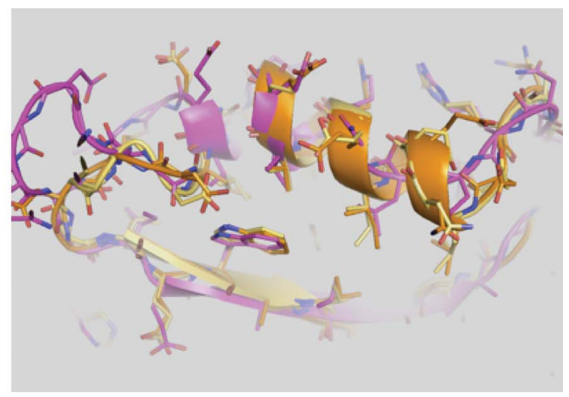

(d)

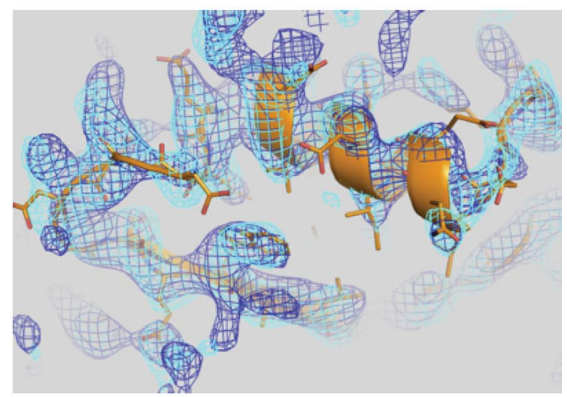

$(f)$

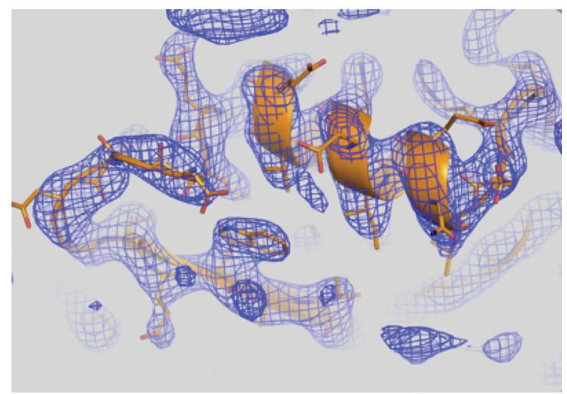

(h)
Figure 5

Comparison of various refinements and maps for residues 251-276. Residues 251-263 comprising an $\alpha$-helix, residues 264-271 comprising a loop and residues 272-276 comprising a $\beta$-strand are shown (the sequence numbers refer to the genomic sequence; see Fig. 1). (a) Standard refinement (gray) versus the final model (orange). Standard refinement produces fragmented or incorrectly connected electron density (marked by arrows). (b) Standard refinement and one round of AutoBuild (blue) versus the final model (orange). Electron density is still fragmented or shows incorrect connectivity. (c) DEN refinement (green) versus the final model (orange). (d) DEN refinement and one round of AutoBuild (magenta) versus the result of semi-automated rebuilding (yellow). (e) $2 m F_{\mathrm{o}}-D F_{\mathrm{c}}$ electron-density map after standard refinement (blue mesh) and a subsequent round of AutoBuild (cyan mesh) versus the final structure (orange sticks). $(f) 2 m F_{\mathrm{o}}-D F_{\mathrm{c}}$ electron-density map after DEN refinement (blue) and a subsequent round of AutoBuild (cyan) versus the final structure (orange sticks). (g) Electron-density map obtained by density modification of the MAD map (blue) versus the final structure (orange sticks). (h) $2 m F_{\mathrm{o}}-D F_{\mathrm{c}}$ electron-density map (blue mesh) of the final model (orange sticks).

\subsection{First round of automated model building with AutoBuild}

Starting from the best DEN-refined structure, automated model building with AutoBuild (Terwilliger et al., 2008) was performed. The default settings for rebuilding the model without the addition or deletion of residues (the rebuild_in_place=true option in AutoBuild) were used except that 'morphing' was enabled and the resolution for multiple model building was set to the limiting resolution of the diffraction data at the inflection-point wavelength $(3.17 \AA)$. The morphing process in AutoBuild consists of identifying a coordinate shift to apply to each backbone $\mathrm{N}$ atom that maximizes the local density correlation between the model and the map (Terwilliger et al., submitted). These coordinate shifts are smoothed and applied to the structure to generate a morphed structure. An initial map was used for AutoBuild consisting of the average of the $2 m F_{\mathrm{o}}-D F_{\mathrm{c}}$ electron-density maps corresponding to the top 20 models (in terms of $R_{\text {free }}$ ) obtained from DEN refinement. Such map averaging can be beneficial (Rice et al., 1998), although in this particular case using the average map was similar to using the map obtained from the top solution. This round of automatic model building produced further improvements in the model (Figs. $4 d$ and $5 d$ ) and lowered the $R$ values $\left(R_{\text {free }}=0.418, R_{\text {cryst }}=0.327\right.$; Table 2).

At this point, it became clear from the electron-density maps produced by DEN refinement $\left(2 m F_{\mathrm{o}}-D F_{\mathrm{c}}\right.$ map) and AutoBuild (both $2 m F_{\mathrm{o}}-D F_{\mathrm{c}}$ and density-modified maps) that the model contained several incorrect sequence registers, resulting in distorted $\alpha$-helices and bulging loops that had no electron density associated with them (a striking example is shown in Fig. 6). DEN refinement and AutoBuild are currently unable to automatically correct such sequence-register shifts and deformed $\alpha$-helices. In particular, AutoBuild has 
no facility for automatic adjustment of sequence register or missing residues when building with the rebuild_in place approach. Still, it was possible to correct these errors by semi-automated rebuilding and manual model building as outlined below. In principle, completely automated rebuilding of the model can be performed for structures at $3 \AA$ resolution (e.g. starting from an experimental electron-density map or a density modified map of a molecular-replacement solution), but for Cgl1109 this approach was not successful, presumably owing to the relatively high anisotropy and $B$ values of the crystal structure. It should be noted that no experimental MAD phase information had been used up to this stage of the refinement process, so it is likely that the structure could have been completed without experimental phase information (Fig. 6).

\subsection{Comparison with standard refinement}

For comparison, we performed 'standard refinement' consisting of three macrocycles of 200 steps of positional (xyz) minimization and 200 steps of restrained individual $B$-factor refinement using $C N S$ starting from the same model that was used for DEN refinement. One round of automated model building starting from this standard refined model was performed using the same options for AutoBuild as for the DEN-refined model (see above).

The $R$ values that were achieved by DEN refinement were significantly lower than those obtained by standard refinement (e.g. $R_{\text {free }}=0.444$ versus 0.517 ; see Table 2). Moreover, the DEN-refined structure was significantly closer to the final model of Cgl1109 (representative examples are shown in Figs. $4 a, 4 c, 5 a$ and $5 c$ ). Automated model building did not significantly improve the model after standard refinement

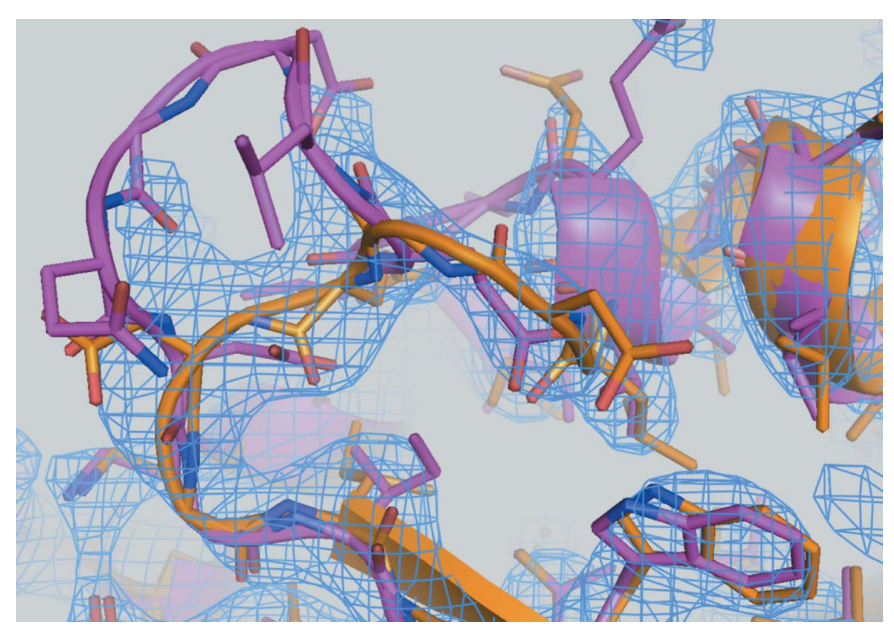

Figure 6

Comparison of various refinements and maps for residues 251-276. A close-up view of the loop consisting of residues 264-271, which is also part of Fig. 5, is shown. The final model is colored orange (sticks and cartoon representation). The structure after the first round of DEN refinement and AutoBuild is colored magenta (sticks and cartoon representation) and the corresponding $2 m F_{\mathrm{o}}-D F_{\mathrm{c}}$ electron-density map (with model phases calculated from this structure, but without experimental phase information, and contoured at $1.4 \sigma$ ) is colored marine blue. The electrondensity map clearly shows that the loop needed to be corrected.
Table 2

$R$ values for different refinement stages and, for comparison, for standard refinement.

\begin{tabular}{lll}
\hline Structure & $R_{\text {free }}$ & $R_{\text {cryst }}$ \\
\hline Phaser solution & - & 0.649 \\
Standard refinement & 0.517 & 0.432 \\
Standard refinement + AutoBuild & 0.483 & 0.374 \\
DEN refinement & 0.444 & 0.399 \\
DEN refinement + AutoBuild & 0.418 & 0.327 \\
Second DEN refinement (MLHL) & 0.397 & 0.366 \\
Second DEN refinement + AutoBuild (MLHL) & 0.372 & 0.325 \\
Final refined & 0.257 & 0.238 \\
\hline
\end{tabular}

(Figs. $4 b$ and $5 b$; Table 2), resulting in $R_{\text {free }}=0.483$ compared with $R_{\text {free }}=0.418$ for the DEN-refined model. This example demonstrates that DEN refinement produces significantly better models than standard refinement for starting models that are far from the true structure, enabling further improvements by automated model building with AutoBuild. In most places there was reasonable agreement between the final model and the $2 m F_{\mathrm{o}}-D F_{\mathrm{c}}$ electron-density maps computed after DEN refinement or subsequent automated model building (Figs. $4 f$ and $5 f$ ). In contrast, the electrondensity maps obtained by standard refinement with and without subsequent automated model building were fragmented and exhibited incorrect connectivity in several places (Figs. $4 e$ and $5 e$ ). Thus, structure completion would have been very difficult to achieve with manual model building and standard refinement.

\subsection{Determination of selenium sites and MAD phasing}

The model obtained from the first round of DEN refinement and automated model building was used to calculate anomalous difference Fourier maps at the peak wavelength $\left(\lambda_{3}\right)$. These difference maps produced difference peaks for the six selenium sites of the SeMet residues in the protein. The positions of these six sites closely matched the positions of the Se atoms in the model obtained after DEN refinement and automated model building. Fig. 7 shows the standard deviations from the mean of the map $(\sigma)$ of these six sites and the highest noise peak. The standard deviations of the peaks are compared with those obtained from standard refinement with and without subsequent automated model building. The combination of DEN refinement and automated model building produced the most significant difference peaks, all of which were well separated from noise. Standard refinement produced the poorest results, with three of the sites close to noise peaks. For both standard refinement and DEN refinement automated model building with AutoBuild improved the significance of the sites, although DEN refinement alone still produced more significant peaks for some of the sites than standard refinement and automated model building. In retrospect, it may have been possible to obtain the positions of the six sites by ab initio search, for example by using the HySS submodule (Grosse-Kunstleve \& Adams, 2003), although careful choice of the high-resolution limit is required (truncation to $4.5 \AA$ resolution) since a search against all diffraction 
data produced only one site that matched one of the six selenium sites.

We next calculated MAD phase probability distributions to $2.97 \AA$ resolution and refined the six selenium sites using a maximum-likelihood method (Burling et al., 1996) as implemented in CNS (Brünger et al., 1998) using the mad_phase.inp task file. The diffraction data collected at the three wavelengths were used (Table 1), anisotropic scale factors between the three data sets were refined, individual $B$ factors for the anomalous sites were refined, occupancies were set to 1 and anomalous form factors were constrained to be identical for all sites at a particular wavelength. The phasing calculations resulted in an overall figure of merit of 0.55 with reasonable overall scale factors, $B$ factors and anomalous form factors of $f^{\prime}=-6.14(-6.95), f^{\prime \prime}=4.73(3.15)$ at the peak, $f^{\prime}=-11, f^{\prime \prime}=5.27$ at the inflection point and $f^{\prime}=-3.32$ $(-3.59), f^{\prime \prime}=3.66(1.05)$ at the remote wavelength, where the numbers refer to the results from the Friedel mate $F$ to $F_{\text {reference }}$ lack-of-closure expressions and the numbers in parentheses refer to the $F$ to $F_{\text {reference }}$ lack-of-closure expressions (Burling et al., 1996). For comparison, the predicted values obtained from a fluorescence scan of the crystal are $f^{\prime}=-8.65, f^{\prime \prime}=6.21$ at the peak, $f^{\prime}=-11.11, f^{\prime \prime}=3.64$ at the inflection point and $f^{\prime}=-1.70, f^{\prime \prime}=3.30$ at the remote wavelength. In our experience, the differences between the refined values of $f^{\prime}$ and $f^{\prime \prime}$ for the two lack-of-closure expressions and from the predicted values are not uncommon for SeMet MAD data.

The resulting MAD electron-density map was subjected to density modification as implemented in CNS (Brünger et al., 1998) using the density_modify.inp task file. The default settings were used, which include solvent flipping with generation of the mask based on root-mean-square electrondensity fluctuations assuming $70 \%$ solvent content. No atomic

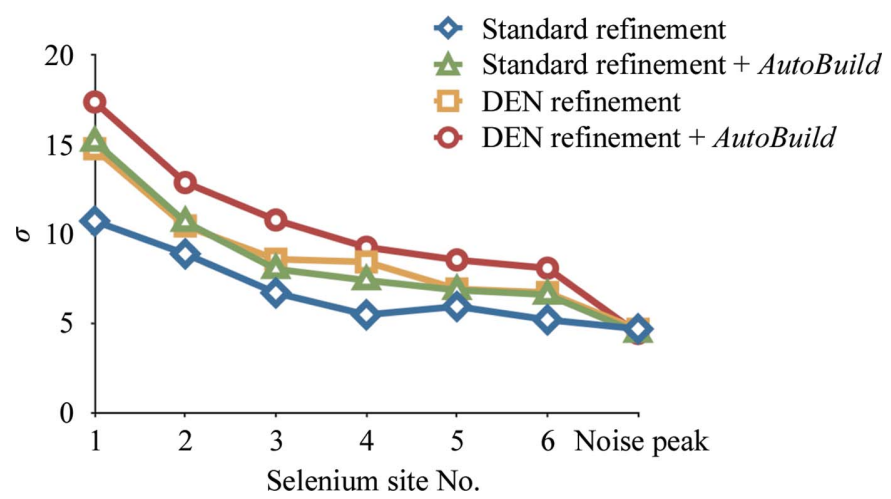

Figure 7

Significance of selenium sites. The standard deviation above the mean $(\sigma)$ in anomalous difference Fourier maps is shown for the six selenium sites of the SeMet variant of Cgl1109. For comparison, the standard deviation of the highest noise peak is also shown. The amplitudes for the calculation of the anomalous difference Fourier map were obtained from the diffraction data at the peak wavelength (Table 1). The phases were obtained from the atomic model after standard refinement (blue diamonds), standard refinement followed by automated building with AutoBuild (green triangles), DEN refinement (yellow squares) and DEN refinement followed by automated model building with AutoBuild (red circles). model was used for the generation of the mask and no prior phase information was used for the refinement of anomalous sites in order to avoid model bias. The resulting figure of merit was 0.81 and the density-modified MAD electron-density map was connected but did not allow unambiguous identification of side chains for many residues (Figs. $4 g$ and $5 g$ ). Although this map may be of sufficient quality such that manual building could have been attempted, it would have been challenging at this resolution. Indeed, automated model building using the same map resulted in a very incomplete model: only 76 side chains were fitted out of 360 , with several false backbone connections.

\subsection{Semi-automated completion of the refinement}

A second round of DEN refinement (using the current model obtained from the first round of DEN refinement and automated model building as both the starting and the reference model) and automated model building was performed using the MLHL target function (Pannu et al., 1998) that included the experimental MAD phase information, resulting in relatively small localized changes in coordinates with some more significant corrections of side-chain positions, improvements in $R$ values and a reduction of the $R_{\text {free }}-R_{\text {cryst }}$ difference (Table 2).

As mentioned above, there were several regions that required correction of register shifts and rebuilding of $\alpha$-helices (a particular example is shown in Fig. 6) that were not corrected even in the second round of DEN refinement and automated model building. To correct these regions, selected regions were deleted from the model and another round of automated rebuilding with AutoBuild was performed, again using the electron-density map from the previous model as the initial map, using the experimental MAD phase information and the primary sequence, with morphing enabled and the rebuild-in-place option set to false. Interestingly, we found that using a $2 m F_{\mathrm{o}}-D F_{\mathrm{c}}$ electron-density map as the initial electron-density map for AutoBuild produced somewhat better results for rebuilding in this particular case than using the density-modified map generated by AutoBuild. The resulting models (using models with different deletions as starting models for automated model building) were inspected using Coot (Emsley et al., 2010) and the portions that best fitted the electron-density maps were combined to generate a hybrid model. Missing loops were fitted with the 'Fit Loops' feature of PHENIX. This procedure of selected rebuilding by deletion of the problematic regions and automated rebuilding was repeated several times. This semi-automated method corrected the majority of cases of incorrectly fitted $\alpha$-helices and loops arising from register errors (Figs. $4 d$ and $5 d$, yellow versus orange models).

The remaining misfitted regions were manually corrected with Coot (Emsley et al., 2010) interspersed with refinement with phenix.refine (Adams et al., 2010). The final refinement (Table 1) employed residues 10-369 of Cgl1109 (a 369-residue protein) and other solvent molecules (one phosphate ion and 
one chloride ion). It was performed against diffraction data collected at the high-energy remote wavelength (Table 1).

\subsection{Biological implications and comparison between 1vgy and Cgl1109}

C. glutamicum is a Gram-positive bacterium that finds industrial use in the production of vitamins and amino acids, including glutamic acid, which is used in the production of the flavoring agent monosodium glutamate. Cgl1109 (NCBI reference sequence identifier NP_600337; UniProt identifier Q59284) is a putative succinyl-diaminopimelate desuccinylase (DapE) from C. glutamicum consisting of two domains: a peptidase domain belonging to family PF01546 (Peptidase M20) in clan CL0035 of zinc metallopeptidases ( 30 000 proteins in 12 families) in v. 25 of the Pfam database (Finn $e t$ al., 2010) and a dimerization domain belonging to PF07687 (M20_dimer). These proteins have a broad phylogenetic spread across all kingdoms of life, show substantial sequence divergence and are essential for numerous biological processes (for example, recombinant bacterial carboxypeptidase G2 is used in cancer therapy to hydrolyze methotrexate and is being tested in prodrug therapy, and human aspartoacylase is implicated in Canavan's disease in the brain), but structural coverage exists for only a small fraction $(\sim 0.3 \%)$ of the proteins in this clan. Cgl1109 was selected by the JCSG to increase the structural coverage of these families and is one of $\sim 20$ structures determined to date (see http:// www.topsan.org/Groups/Zinc_Peptidase). DapE is involved in producing L-lysine and L,L-2,6-diaminopimelate and its catalytic mechanism is likely to involve two zinc ions.

The crystal structure of Cgl1109 reveals a dimeric structure from crystal-packing considerations and as also suggested by the PDBePISA server (Fig. 2). The dimeric assembly is promoted by the smaller of the two domains of the molecule (Fig. 8), while the larger domain is the putative catalytic domain. The dimeric assembly is consistent with proteins from this family that contain a similar dimerization domain. Electron density in $2 m F_{\mathrm{o}}-D F_{\mathrm{c}}$ and $m F_{\mathrm{o}}-D F_{\mathrm{c}}$ maps initially suggested the possible presence of two zinc ions in the putative catalytic site, which would be expected owing to the addition of $6 \mathrm{mM} \mathrm{ZnCl}$ during cocrystallization (which was added based on putative functional annotation and ligand screening in a fluorescence-based thermal shift assay). However, we did not model zinc ions in the final model owing to the uncertainty associated with high $B$ factors and the absence of significant peaks in the anomalous difference Fourier maps, including from diffraction data collected at the zinc absorption edge.

Fig. 8 shows a superposition of Cgl1109 with the template used for homology modeling (PDB entry 1vgy, chain $A$ ), a putative succinyl-diaminopimelate desuccinylase from $N$. meningitidis. The superposition shows that the overall fold is identical, but that there are large differences in secondarystructural element placement and length, as perhaps expected considering the low sequence identity $(25 \%)$ between the proteins and the resulting difficulties with molecularreplacement phasing.

\section{Conclusions}

Successful structure determination of the difficult molecularreplacement example Cgl1109 illustrates the synergism between DEN refinement and automated model building with AutoBuild. DEN refinement is most beneficial at the early stage of the refinement process, immediately after molecularreplacement phasing, when the model is still relatively crude and distant from the true structure. For Cgl1109, DEN refinement resulted in a model that is closer to the true structure, producing improved model phases that in turn provide a better starting point for automated model building. The improved model phases also provided more significant peaks in anomalous difference Fourier maps to better locate the six selenium sites of the protein. In contrast, standard refinement (i.e. positional and $B$-factor refinement) produced fragmented electron density with incorrect connectivity (marked by arrows in Fig. 5e). The $R$ values that we obtained after the initial round of DEN refinement and automated model building with AutoBuild are better than those reported in Table 1 of DiMaio et al. (2011) ( $R_{\text {free }}=0.418$ versus $\left.R_{\text {free }}=0.460\right)$. This difference is most likely to arise from performing a full $\left(\gamma, w_{\text {DEN }}\right)$ grid search with multiple repeats with different initial velocities and random selection of DEN restraints at each grid point in the present work as opposed to a single DEN refinement as was performed previously (DiMaio et al., 2011). Our success in fully refining the Cgl1109 structure also demonstrates that the combination of DEN refinement and automated model building is a viable alternative to the Rosetta molecular-replacement approach (DiMaio et al., 2011). However, further analysis is required to determine the optimal application and potential limitations of both methods.

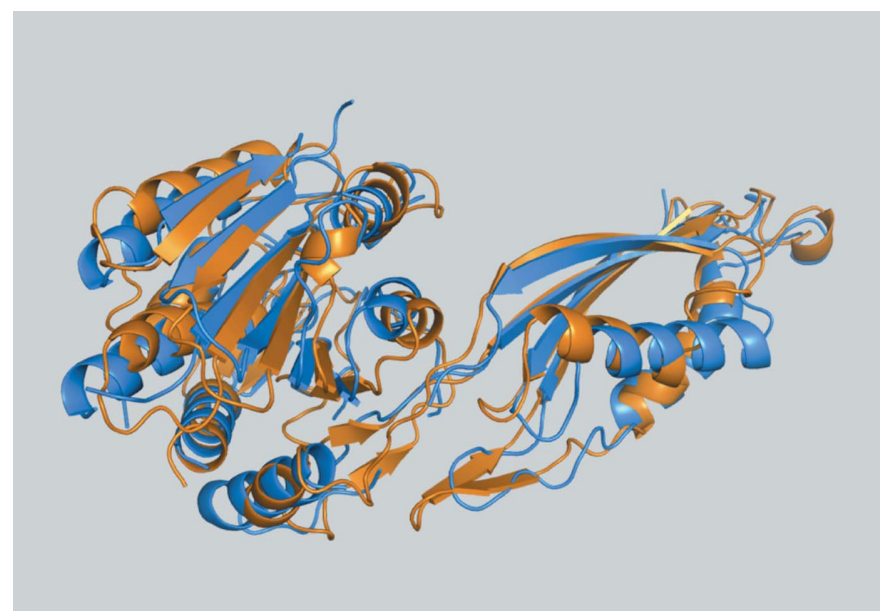

Figure 8

Comparison of Cgl1109 with 1vgy- $A$. A superposition of the final model of Cgl1109 (orange cartoon) and chain $A$ of PDB entry 1vgy (blue cartoon) is shown. The superposition was performed with PyMOL (DeLano, 2002). 
Poorly fitted portions of the model after DEN refinement and automated model building were readily identified by inspection of the electron-density maps (Fig. 6). These electron-density maps unambiguously suggested how to correct the model. It turned out that most of these regions were related to local sequence misalignments. We generated a structure-based alignment between the template 1vgy- $A$ and Cgl1109 using MUSTANG (Konagurthu et al., 2006) and compared it with predicted alignments. PROMALS3D and HHpred correctly assigned 282 and 291 positions (of a total of 360 residues visible in the $\mathrm{Cgl1109}$ structure), respectively. The difference between the PROMALS3D and HHpred alignments is caused by a one-register shift involving an $\alpha$-helix (residues 132-140). This one-residue shift required manual rebuilding when using the PROMALS3D alignment for the molecular-replacement search model. In retrospect, it might have been beneficial to use models generated by both the PROMALS3D and HHpred alignments as starting points for DEN refinement and automated model building and then to generate a composite model keeping the best-fitting parts of both models.

Sequence-register errors that arise from local misalignments between the target protein and the homology model can be difficult to correct using automated model-building methods when working with electron-density maps at low resolution or those based on highly anisotropic diffraction data. Overinterpretation or misinterpretation of such lowresolution maps is a real danger when they are manually interpreted without assistance from more objective computational methods. Indeed, we were able to partially automate the process by deleting the incorrectly aligned regions and rebuilding the parts with automated methods; some remaining regions had to be manually corrected. In particular, AutoBuild will sometimes misfit $\alpha$-helices at low resolution, tracing the chain through the center of the $\alpha$-helix (Fig. $5 d$, magenta). It should be noted, however, that in this case the method of deleting the $\alpha$-helix from the current model and rebuilding it from scratch produced the correct fit (Fig. 5d, yellow). However, in two other instances this approach was not successful and the $\alpha$-helices had to be manually rebuilt. It seems possible that this process could be fully automated. This would be especially important for low-resolution structures, in which interpretation of the electron-density map by inspection can be subjective and can lead to local misfitting (DeLaBarre \& Brunger, 2005; Davies et al., 2008). It is conceivable that a systematic method to probe the fit with different local sequence alignments in problematic regions might produce the best possible model for such low-resolution structures.

Genomic DNA from C. glutamicum 534 (ATCC No. 13032D) was obtained from the American Type Culture Collection (ATCC). We thank all members of the JCSG for their contribution to the development and operation of our HTP structural biology pipeline and for bioinformatics analysis, protein production and structure determination. The JCSG is supported by the NIH, National Institutes of General
Medical Sciences, Protein Structure Initiative (U54 GM094586 and GM074898). Portions of this research were performed at the Stanford Synchrotron Radiation Lightsource (SSRL), SLAC National Accelerator Laboratory. The SSRL is a national user facility operated by Stanford University on behalf of the United States Department of Energy, Office of Basic Energy Sciences. The SSRL Structural Molecular Biology Program is supported by the Department of Energy, Office of Biological and Environmental Research and by the National Institutes of Health (National Center for Research Resources, Biomedical Technology Program and the National Institute of General Medical Sciences). ATB acknowledges funding by the Howard Hughes Medical Institute. RJR is supported by the Wellcome Trust. ML acknowledges NIH grant GM063817. This work was supported in part by the US Department of Energy under contract No. DE-AC0376SF00098 at Lawrence Berkeley National Laboratory and NIH/NIGMS grant 1P01GM063210 to PDA, RJR and TCT. The content is solely the responsibility of the authors and does not necessarily represent the official views of the National Institute of General Medical Sciences or the National Institutes of Health.

\section{References}

Adams, P. D. et al. (2010). Acta Cryst. D66, 213-221.

Afonine, P. V., Grosse-Kunstleve, R. W. \& Adams, P. D. (2005). CCP4 Newsl. Protein Crystallogr. 41, contribution 8.

Badger, J. et al. (2005). Proteins, 60, 787-796.

Brünger, A. T., Adams, P. D., Clore, G. M., DeLano, W. L., Gros, P., Grosse-Kunstleve, R. W., Jiang, J.-S., Kuszewski, J., Nilges, M., Pannu, N. S., Read, R. J., Rice, L. M., Simonson, T. \& Warren, G. L. (1998). Acta Cryst. D54, 905-921.

Bunkóczi, G. \& Read, R. J. (2011). Acta Cryst. D67, 303-312.

Burling, F. T., Weis, W. I., Flaherty, K. M. \& Brünger, A. T. (1996). Science, 271, 72-77.

Chen, V. B., Arendall, W. B., Headd, J. J., Keedy, D. A., Immormino, R. M., Kapral, G. J., Murray, L. W., Richardson, J. S. \& Richardson, D. C. (2010). Acta Cryst. D66, 12-21.

Cohen, A. E., Ellis, P. J., Miller, M. D., Deacon, A. M. \& Phizackerley, R. P. (2002). J. Appl. Cryst. 35, 720-726.

Cowtan, K. (2006). Acta Cryst. D62, 1002-1011.

D'Arcy, A., Villard, F. \& Marsh, M. (2007). Acta Cryst. D63, 550-554.

Davies, J. M., Brunger, A. T. \& Weis, W. I. (2008). Structure, 16, 715-726.

DeLaBarre, B. \& Brunger, A. T. (2005). J. Mol. Biol. 347, 437-452.

DeLano, W. L. (2002). PyMOL. http://www.pymol.org.

DePristo, M. A., de Bakker, P. I., Johnson, R. J. \& Blundell, T. L. (2005). Structure, 13, 1311-1319.

Diederichs, K. \& Karplus, P. A. (1997). Nature Struct. Biol. 4, 269-275.

DiMaio, F., Terwilliger, T. C., Read, R. J., Wlodawer, A., Oberdorfer, G., Wagner, U., Valkov, E., Alon, A., Fass, D., Axelrod, H. L., Das, D., Vorobiev, S. M., Iwaï, H., Pokkuluri, P. R. \& Baker, D. (2011). Nature (London), 473, 540-543.

Elsliger, M.-A., Deacon, A. M., Godzik, A., Lesley, S. A., Wooley, J., Wüthrich, K. \& Wilson, I. A. (2010). Acta Cryst. F66, 1137-1142.

Emsley, P., Lohkamp, B., Scott, W. G. \& Cowtan, K. (2010). Acta Cryst. D66, 486-501.

Finn, R. D., Mistry, J., Tate, J., Coggill, P., Heger, A., Pollington, J. E., Gavin, O. L., Gunasekaran, P., Ceric, G., Forslund, K., Holm, L., Sonnhammer, E. L., Eddy, S. R. \& Bateman, A. (2010). Nucleic Acids Res. 38, 211-222.

Grosse-Kunstleve, R. W. \& Adams, P. D. (2003). Acta Cryst. D59, 1966-1973. 
Ioerger, T. R. \& Sacchettini, J. C. (2003). Methods Enzymol. 374, 244-270.

Ireton, G. C. \& Stoddard, B. L. (2004). Acta Cryst. D60, 601-605.

Kabsch, W. (2010). Acta Cryst. D66, 125-132.

Kleywegt, G. J. (2000). Acta Cryst. D56, 249-265.

Klock, H. E., Koesema, E. J., Knuth, M. W. \& Lesley, S. A. (2008). Proteins, 71, 982-994.

Konagurthu, A. S., Whisstock, J. C., Stuckey, P. J. \& Lesk, A. M. (2006). Proteins, 64, 559-574.

Langer, G., Cohen, S. X., Lamzin, V. S. \& Perrakis, A. (2008). Nature Protoc. 3, 1171-1179.

Levitt, D. G. (2001). Acta Cryst. D57, 1013-1019.

McCoy, A. J., Grosse-Kunstleve, R. W., Adams, P. D., Winn, M. D., Storoni, L. C. \& Read, R. J. (2007). J. Appl. Cryst. 40, 658-674.

McPhillips, T. M., McPhillips, S. E., Chiu, H.-J., Cohen, A. E., Deacon, A. M., Ellis, P. J., Garman, E., Gonzalez, A., Sauter, N. K., Phizackerley, R. P., Soltis, S. M. \& Kuhn, P. (2002). J. Synchrotron Rad. 9, 401-406.

Murshudov, G. N., Skubák, P., Lebedev, A. A., Pannu, N. S., Steiner, R. A., Nicholls, R. A., Winn, M. D., Long, F. \& Vagin, A. A. (2011). Acta Cryst. D67, 355-367.

Oldfield, T. J. (2002). Acta Cryst. D58, 487-493.

Oldfield, T. (2003). Acta Cryst. D59, 483-491.

Pannu, N. S., Murshudov, G. N., Dodson, E. J. \& Read, R. J. (1998). Acta Cryst. D54, 1285-1294.
Pannu, N. S. \& Read, R. J. (1996). Acta Cryst. A52, 659668.

Pei, J., Kim, B.-H. \& Grishin, N. V. (2008). Nucleic Acids Res. 36, 2295-2300.

Rice, L. M., Shamoo, Y. \& Brünger, A. T. (1998). J. Appl. Cryst. 31, 798-805.

Sali, A. \& Blundell, T. L. (1993). J. Mol. Biol. 234, 779-815.

Schröder, G. F., Levitt, M. \& Brunger, A. T. (2010). Nature (London), 464, 1218-1222.

Schwarzenbacher, R., Godzik, A., Grzechnik, S. K. \& Jaroszewski, L. (2004). Acta Cryst. D60, 1229-1236.

Söding, J. (2005). Bioinformatics, 21, 951-960.

Terwilliger, T. C. (2000). Acta Cryst. D56, 965-972.

Terwilliger, T. C., Grosse-Kunstleve, R. W., Afonine, P. V., Moriarty, N. W., Zwart, P. H., Hung, L.-W., Read, R. J. \& Adams, P. D. (2008). Acta Cryst. D64, 61-69.

Thompson, J. D., Higgins, D. G. \& Gibson, T. J. (1994). Nucleic Acids Res. 22, 4673-4680.

Vaguine, A. A., Richelle, J. \& Wodak, S. J. (1999). Acta Cryst. D55, 191-205.

Van Duyne, G. D., Standaert, R. F., Karplus, P. A., Schreiber, S. L. \& Clardy, J. (1993). J. Mol. Biol. 229, 105-124.

Vriend, G. (1990). J. Mol. Graph. 8, 52-56.

Yang, H., Guranovic, V., Dutta, S., Feng, Z., Berman, H. M. \& Westbrook, J. D. (2004). Acta Cryst. D60, 1833-1839. 